\title{
Image Alignment Using 2D Interpolation in the Frequency Domain for Class Averaging in Single- particle Cryo-EM
}

\section{Xiangwen Wang}

Lanzhou University

Yonggang Lu ( $\nabla$ ylu@lzu.edu.cn )

Lanzhou University

Jiaxuan Liu

Lanzhou University

\section{Research Article}

Keywords: Cryo-electron microscopy, Single-particle reconstruction, Class averaging, Image alignment, 2D interpolation

Posted Date: May 21st, 2021

DOl: https://doi.org/10.21203/rs.3.rs-515809/v1

License: (c) (i) This work is licensed under a Creative Commons Attribution 4.0 International License. Read Full License 


\section{Image Alignment Using 2D Interpolation in the Frequency Domain for Class Averaging in Single-Particle Cryo-EM}

\author{
${ }^{*}$ Correspondence: ylu@lzu.edu.cn \\ ${ }^{1}$ School of Information Science \\ and Engineering, Lanzhou \\ University, 730000, Lanzhou, \\ China \\ Full list of author information is \\ available at the end of the article
}

Xiangwen Wang ${ }^{1,2}$, Yonggang $\mathrm{Lu}^{1 *}$ and Jiaxuan $\mathrm{Liu}^{1}$

\begin{abstract}
Background: Three-dimensional (3D) reconstruction in single-particle cryo-electron microscopy is a significant technique for recovering the 3D structure of proteins or other biological macromolecules from their two-dimensional (2D) noisy projection images taken from unknown random directions. Class averaging in single-particle cryo-electron microscopy is an important procedure for producing high-quality initial 3D models due to the existence of a high level of noise in the projection images. Image alignment is a fundamental step in the class averaging.
\end{abstract}

Results: In this paper, an efficient image alignment algorithm using 2D interpolation in the frequency domain of images is proposed to improve the estimation accuracy of alignment parameters. The proposed algorithm firstly uses the Fourier transform of two projection images to calculate a discrete cross-correlation matrix and then performs the $2 \mathrm{D}$ interpolation around the maximum value in the cross-correlation matrix. The alignment parameters of rotation angles and translational shifts in the $x$-axis and $y$-axis directions between the two projection images are directly determined according to the position of the maximum value in the cross-correlation matrix after interpolation. Furthermore, the proposed algorithm and the K-medoids clustering algorithm are used to compute class averages for single-particle 3D reconstruction.

Conclusions: Results on simulated data set show that the proposed algorithm can be used to compute the alignment parameters efficiently, and using the 2D interpolation can improve the estimation accuracy of the alignment parameters, which usually leads to a better $3 \mathrm{D}$ reconstruction result.

Keywords: Cryo-electron microscopy; Single-particle reconstruction; Class averaging; Image alignment; 2D interpolation

\section{Introduction}

Cryo-electron microscopy (cryo-EM) has become a recognized powerful technique in structural biology for 3D structure determination of biological macromolecules, supramolecular complexes, and subcellular structures $[1,2,3]$. It does not need crystallization and has been widely used to study large macromolecular complexes that are difficult to be crystallized. The goal of cryo-EM 3D reconstruction is to reconstruct a high-resolution estimation of the 3D electron density map of the molecule from a set of micrographs $[4,5,6]$. Cryo-EM can be used to investigate complete and fully functional macromolecular complexes in different functional states, providing a richness of biological insight $[7,8]$. Cryo-EM has made tremendous progress 
in the past few years $[9,10]$. Owing to these exciting new developments, cryo-EM was selected by Nature Methods as the "Method of the Year 2015", and the Nobel Prize in Chemistry 2017 was awarded to Jacques Dubochet, Joachim Frank, and Richard Henderson "for developing cryo-electron microscopy for the high-resolution structure determination of biomolecules in solution" [5].

As one of the major cryo-EM techniques, single-particle reconstruction has become one of the most successful techniques for structural biology $[11,12,13]$. Singleparticle reconstruction using cryo-EM has been undergoing fast transformations, leading to an abundance of new high-resolution structures and reaching close to atomic resolution $[14,15]$. In the single-particle reconstruction, the same macromolecule is projected from various unknown directions, and the final 3D structure of the macromolecule can be reconstructed from the projection images of the single particles with different estimated projection directions in $3 \mathrm{D}$ space $[16,17]$. One of the major challenges to be overcome in the single-particle reconstruction of biological samples is to estimate the projection directions of the projection images $[18,19]$. However, due to the very low signal-to-noise ratio (SNR) of the projection images caused by low-dose electron radiation, it is usually difficult to get the correct estimation of the projection directions. Consequently, the single-particle 3D reconstruction of cryo-EM is a very challenging task [20, 21].

Class averaging in single-particle cryo-EM is an important procedure for producing high-quality initial 3D models and discarding invalid particles or contaminants [22]. It organizes a data set by grouping together the particles corresponding to the same (or quite similar) projection directions. Each group of cryo-EM projection images is regarded as a class and is averaged to produce an averaged image called a class average. By averaging, the random noise in the background tends to cancel, and the features of interest in the projection images reinforce each other as the number of superimposed projection images becomes large [23, 24]. Class averages can be used to improve ab initio modelling in cryo-EM. They can also be applied for discovering heterogeneity or symmetricity as well as for separating particles into subgroups for additional analysis [25].

Image alignment is a fundamental step in the class averaging procedure [26, 27]. The cryo-EM projection images are required to be identified and rotationally and translationally aligned to distinguish among different classes. After alignment, the cryo-EM projection images with nearly the same projection directions are grouped in the 2D classification step. Well-aligned cryo-EM projection images with correct in-plane rotations and translational shifts in the $\mathrm{x}$-axis and $\mathrm{y}$-axis directions can improve the accuracy of the 2D classification [28]. Correctly classify the cryo-EM projection images into homogeneous groups renders the satisfactory determination of the preliminary 3D structures [29]. Although translational invariant and rotational invariant image representation methods have been used in cryo-EM, they usually are not powerful enough to discover subtle differences between projection images [30]. It is necessary to design efficient image alignment algorithms to find the best alignment parameters and generate high-quality class averages.

Different solutions have been proposed for the 2D class averaging problem in cryo-EM [31, 32, 33, 34, 35, 36]. Some popular cryo-EM software packages, such as Xmipp [37, 38, 39], EMAN [40, 41], cryoSPARC [42], cisTEM [43], and RELION $[44,45,46]$ have implemented $2 \mathrm{D}$ class averaging. RELION uses a maximum 
likelihood expectation maximization (ML-EM) 2D classification procedure to infer parameters for a statistical model from the data. The ML-EM scheme has suffered less from initial reference bias, but it is computationally expensive. The iterative stable alignment and clustering (ISAC) algorithm [47] is another famous 2D class averaging method. ISAC relies on a modified K-means clustering method and the concepts of stability and reproducibility, which can extract validated, homogeneous subsets of projection images. ISAC is also time-consuming.

In order to obtain high-resolution 3D structures of biological macromolecules, the procedure of single-particle reconstruction of cryo-EM is usually split into two stages: constructing a preliminary low-resolution model (initial model), which is later refined by applying an iterative refinement algorithm. This paper is focused on the preliminary model reconstruction using $2 \mathrm{D}$ class averages. The single-particle projection images are firstly aligned and then classified into different groups. The projection images in each group are averaged to generate a class average, and the projection directions of each class average are computed. Image alignment is a fundamental step in this series of operations. Image rotational alignment and translational alignment in real space need too many iterations to compute the alignment parameters, and the calculated alignment parameters are integers. In Fourier space, alignment parameters of rotation angles and translational shifts in the $\mathrm{x}$-axis and $\mathrm{y}$-axis directions can be computed directly without enumeration.

Image alignment is aimed at estimating three alignment parameters: rotation angle and two translational shifts in the x-axis and y-axis directions. In this paper, an efficient image alignment algorithm using the $2 \mathrm{D}$ interpolation in the frequency domain of images is proposed to improve the estimation accuracy of alignment parameters. Specifically: (1) for image rotational alignment, two images are transformed by polar fast Fourier transform (PFFT) to calculate a discrete cross-correlation matrix, and then the $2 \mathrm{D}$ interpolation is performed around the maximum value in the cross-correlation matrix. The rotation angle between the two images is directly determined according to the position of the maximum value in the cross-correlation matrix after interpolation. (2) For image translational alignment, all operation steps are consistent with image rotational alignment, where fast Fourier transform (FFT) is used instead of PFFT. (3) For image alignment with rotation and translation, only a few iterations of combined rotational and translational alignment are needed to align images. Furthermore, the proposed algorithm and the K-medoids clustering algorithm [48] instead of K-means clustering are used to compute class averages for single-particle 3D reconstruction. The main contributions of this paper are summarized as follows:

- 2D interpolation in the frequency domain is used to improve the estimation accuracy of the alignment parameters.

- The alignment parameters of rotation angles and translational shifts in the $\mathrm{x}$-axis and $\mathrm{y}$-axis directions can be computed directly in Fourier space without enumeration.

- The K-medoids clustering algorithm is used for the unsupervised 2D classification of single-particle cryo-EM projection images.

The rest of this paper is organized as follows. In section 2, the proposed image alignment algorithm is described in detail, including the image rotational alignment 
and the image translational alignment. In section 3, the flexibility and performance of the proposed algorithm are demonstrated through simulated data. Finally, this paper is concluded in section 4 .

\section{Materials and methods}

In this section, the proposed image alignment algorithm is demonstrated in detail, including (1) image rotational alignment; (2) image translational alignment; (3) image alignment with rotation and translation. Then the proposed algorithm and the K-medoids clustering algorithm are used to compute class averages.

\section{Image rotational alignment}

Image rotational alignment is one of the basic operations in image processing. The rotation angle between two images can be estimated either in real space or in Fourier space. In real space, image rotational alignment is a rotation-matching process, that is, an exhaustive search. An image is rotated in a certain step size, and the similarity between the rotated image and the reference image is calculated. When the image is rotated for one circle, the index corresponding to the maximum similarity is the final estimated rotation angle between the two images. This method is simple, but it is time-consuming and inaccurate. Assuming the search step size is $p$, image rotational alignment in real space requires $360 / p$ rotation-matching calculations. Although the coarse-to-fine search method can be used, it still needs to be calculated many times.

In this paper, the image rotational alignment is implemented in Fourier space without rotation-matching iteration, which is a direct calculation method. In general, the cryo-EM projection images are square, so only the rotational alignment of the square image is considered. For two images $M_{1}$ and $M_{2}$ of size $m * m$, the proposed image rotational alignment method is illustrated in Fig. 1. In the rest of this paper, the proposed image rotational alignment algorithm is represented as function $\operatorname{rot} \operatorname{Align}(\cdot, \cdot)$. There are three key steps in the image rotational alignment algorithm:

- Step 1: Calculate a cross-correlation matrix using PFFT. Firstly, images $M_{1}$ and $M_{2}$ are transformed by PFFT to obtain two corresponding spectrum maps $F_{1}$ and $F_{2}$ with the size of $\lfloor m / 2\rfloor * 360$. Then the cross-correlation matrix $C$ is calculated according to:

$$
C=\operatorname{abs}\left(\text { ifft2 }\left(F_{1} \times \operatorname{conj}\left(F_{2}\right)\right)\right)
$$

where $\operatorname{abs}(\cdot)$ is an absolute value function, if $f t 2(\cdot)$ is a $2 \mathrm{D}$ inverse fast Fourier transform function, and $\operatorname{conj}(\cdot)$ is a complex conjugate function. These functions have been implemented in MATLAB. The values in matrix $C$ need to be circularly shifted by $\lfloor m / 4\rfloor$ positions to exchange rows to horizontally center the large values in matrix $C$, where the function circshift implemented in MATLAB can be used. The size of the cross-correlation matrix $C$ is $\lfloor m / 2\rfloor * 360$.

- Step 2: 2D interpolation around the maximum value in the cross-correlation matrix $C$. The rotation angle $\delta \theta$ of the image $M_{2}$ relative to the image $M_{1}$ can be roughly determined according to the position of the maximum value 


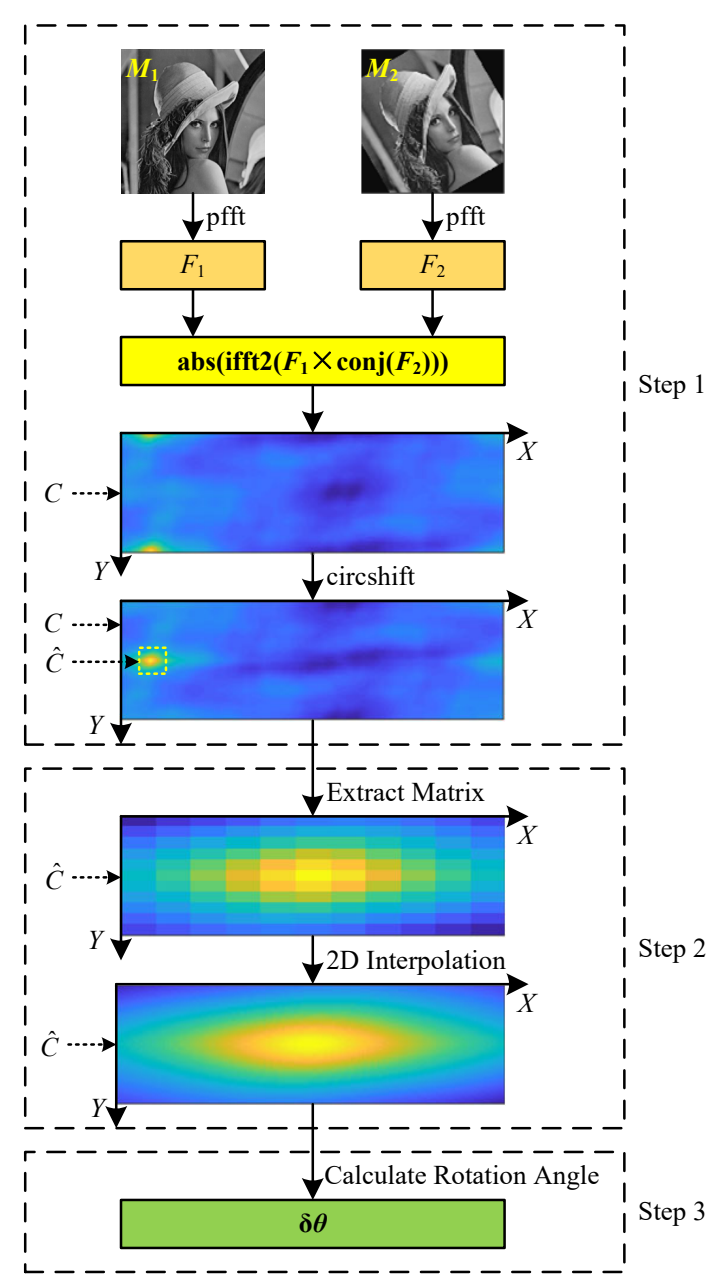

Figure $1 \mathrm{~A}$ diagram of the proposed image rotational alignment algorithm using 2D interpolation in the frequency domain of images.

in the cross-correlation matrix $C$ on the x-axis. The rotation angle calculated by this method is an integer. In order to calculate the rotation angle more accurately, the $2 \mathrm{D}$ interpolation is performed around the maximum value in the cross-correlation matrix $C$. Specifically, an $11^{*} 11$ matrix $\hat{C}$ centered on the maximum value in the matrix $C$ is extracted from the matrix $C$ (see the dotted box in Fig. 1), and then the 2D interpolation is performed in the matrix $\hat{C}$. After 2 D interpolation, the size of the matrix $\hat{C}$ becomes $101 * 101$.

- Step 3: Calculate the rotation angle. The rotation angle $\delta \theta$ can be directly calculated according to the position of the maximum value in the matrix $\hat{C}$ after interpolation on the x-axis. Generally, the rotation angle $\delta \theta$ of an image is in the range of $\left[-180^{\circ}, 180^{\circ}\right]$, so $\delta \theta$ needs to be corrected according to:

$$
\delta \theta=\left\{\begin{array}{lll}
\delta \theta, & \text { if } \quad 0^{\circ} \leq \delta \theta \leq 180^{\circ} \\
\delta \theta-360^{\circ}, & \text { if } \quad 180^{\circ}<\delta \theta<360^{\circ}
\end{array}\right.
$$




\section{Image translational alignment}

Image translational alignment can also be realized in real space or Fourier space. In real space, image translational alignment is also an exhaustive search, and it is more complex than image rotational alignment. For two images $M_{1}$ and $M_{2}$ of size $m * m$, it needs to compute the similarity between each row (column) of $M_{1}$ and each row (column) of $M_{2}$, and then determines the translational shift $\delta x$ in the $\mathrm{x}$-axis direction and the translational shift $\delta y$ in the $\mathrm{y}$-axis direction according to the maximum similarity. Therefore, image translational alignment in real space requires $2 * m * m$ similarity calculations. Also, the translational shifts estimated in real space are integers, which are not accurate enough.

Similar to image rotational alignment, in this paper, the image translational alignment is implemented in Fourier space. It is a direct calculation method without enumeration. For two images $M_{1}$ and $M_{2}$ of size $m * m$, the proposed image translational alignment method is illustrated in Fig. 2. In the rest of this paper, the proposed image translational alignment algorithm is represented as function shiftAlign $(\cdot, \cdot)$. There are three key steps in the image translational alignment algorithm:

- Step 1: Calculate a cross-correlation matrix using FFT instead of PFFT. Firstly, images $M_{1}$ and $M_{2}$ are transformed by FFT to obtain two corresponding spectrum maps $F_{1}$ and $F_{2}$ with size of $m * m$. Then the cross-correlation matrix $C$ is calculated according to:

$$
C=\operatorname{ifft2}\left(F_{1} \times \operatorname{conj}\left(F_{2}\right)\right)
$$

The values in matrix $C$ need to be shifted to center the large values in matrix $C$, where the function $f f t s h i f t$ implemented in MATLAB can be used. The size of the cross-correlation matrix $C$ is $m * m$.

- Step 2: 2D interpolation around the maximum value in the cross-correlation matrix $C$. The translational shifts $\delta x$ and $\delta y$ of the image $M_{2}$ relative to the image $M_{1}$ in the x-axis and y-axis directions can be roughly determined according to the position $(x, y)$ of the maximum value in the cross-correlation matrix $C$ on the $\mathrm{x}$-axis and $\mathrm{y}$-axis, respectively. The translational shifts calculated by this method are integers. In order to calculate the translational shifts more accurately, same as the image rotational alignment described in Section 2.1, the 2D interpolation is performed around the maximum value in the cross-correlation matrix $C$. Specifically, an $11^{*} 11$ matrix $\hat{C}$ centered on the maximum value in the matrix $C$ is extracted from the matrix $C$ (see the dotted box in Fig. 2), and then the 2D interpolation is performed in the matrix $\hat{C}$. After 2D interpolation, the size of the matrix $\hat{C}$ becomes $101^{*} 101$.

- Step 3: Calculate the translational shifts. The translational shifts $\delta x$ and $\delta y$ of the image $M_{2}$ relative to the image $M_{1}$ are directly calculated according to the position $(x, y)$ of the maximum value in the matrix $\hat{C}$ after interpolation on the $\mathrm{x}$-axis and $\mathrm{y}$-axis, respectively:

$$
\left\{\begin{array}{l}
\delta x=\lfloor m / 2\rfloor-x+1 \\
\delta y=\lfloor m / 2\rfloor-y+1
\end{array}\right.
$$




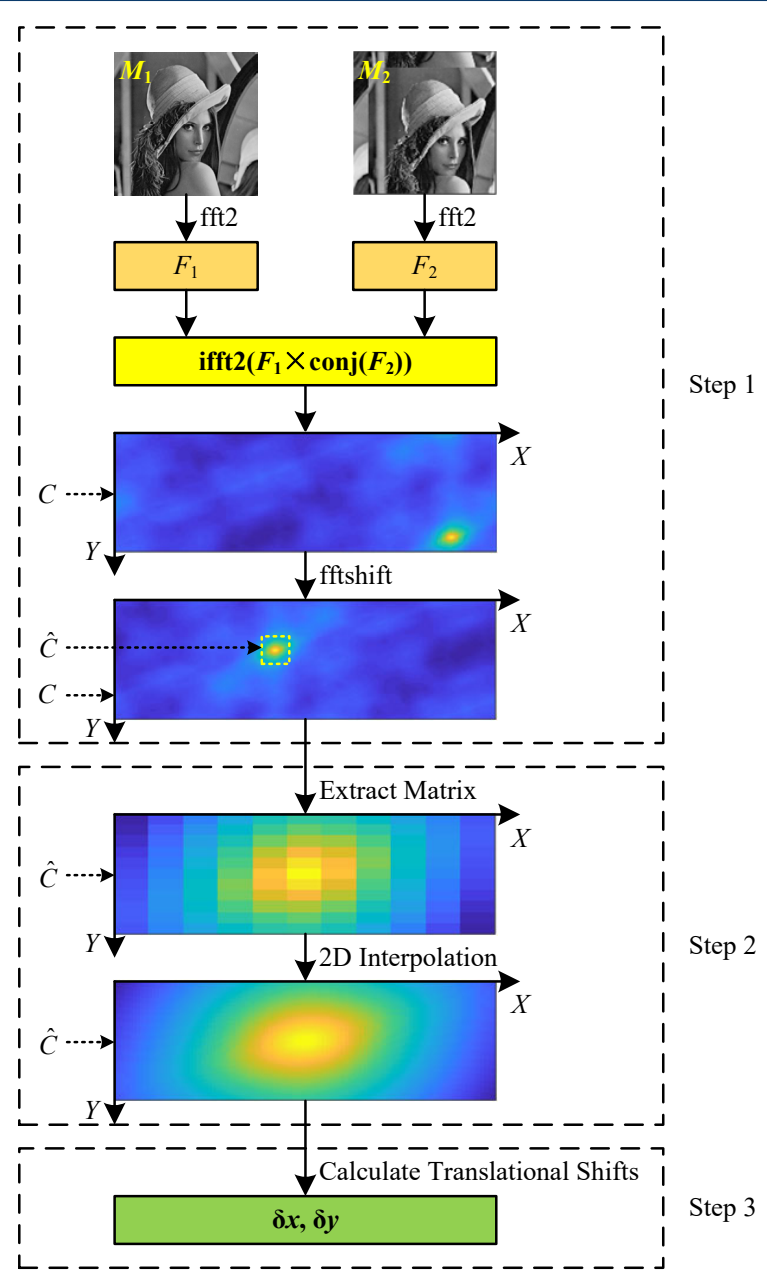

Figure 2 A diagram of the proposed image translational alignment algorithm using 2D interpolation in the frequency domain of images.

Image alignment with rotation and translation

Image alignment with rotation and translation is a fundamental but challenging step in class averaging. It is the coupling of image rotational alignment and image translational alignment and generally requires iterations. In this paper, an efficient image alignment algorithm using the 2D interpolation in the frequency domain of images is proposed, which is listed in Algorithm 1. Functions $\operatorname{rotAlign}(\cdot, \cdot)$ and $\operatorname{shiftAlign}(\cdot, \cdot)$ represent the image rotational alignment algorithm described in Section 2.1 and the image translational alignment algorithm described in Section 2.2 , respectively. Functions imrotate $(\cdot, \cdot)$ and $\operatorname{imshift}(\cdot, \cdot)$ represent the image rotation operation and image translation operation, respectively. For each iteration, the test image $M$ is first rotationally aligned and then translationally aligned to calculate three alignment parameters. The final alignment parameters $\Delta \theta, \Delta x, \Delta y$ and the original test image $M$ are used to calculate the final aligned image $M_{A}$, reducing the error accumulation caused by interpolation calculation in image rotation and translation. When the alignment parameters $\delta \theta, \delta x, \delta y$ during the iteration remain unchanged, the iteration can be terminated ahead of time. According to our 
experience, the program will converge within ten iterations in the majority of cases. In addition, there is no complicated operation in the algorithm. It can efficiently and effectively align two images. Therefore, the proposed algorithm can be used to align a large number of cryo-EM projection images.

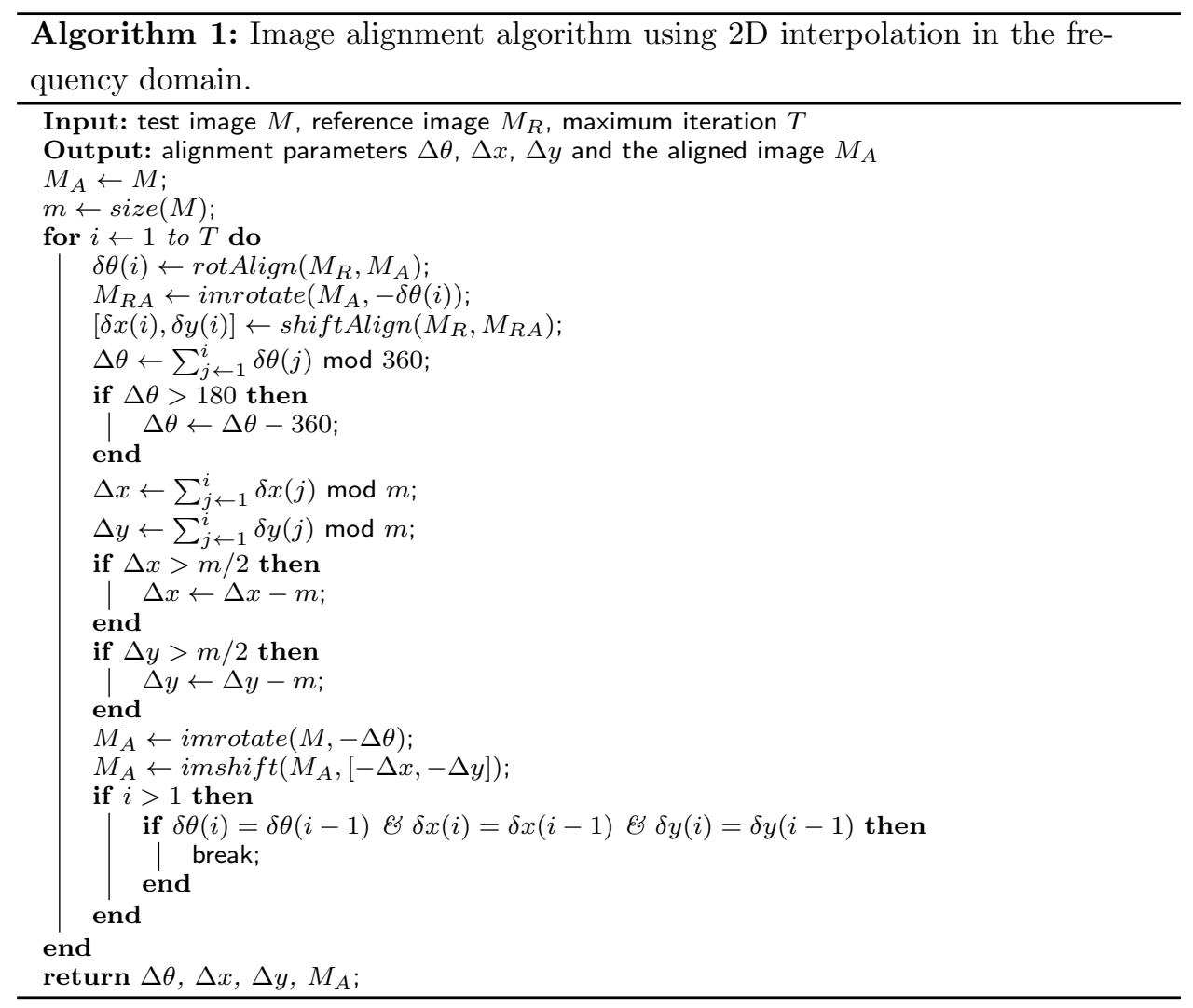

\section{Class averaging}

In this paper, the proposed image alignment algorithm and the K-medoids clustering algorithm are used to implement class averaging. The calculation process of the class averaging is shown in Fig. 3. First of all, all the cryo-EM projection images are aligned by the proposed image alignment algorithm to calculate the distance between them, where the $2 \mathrm{D}$ correlation coefficient is used. The $2 \mathrm{D}$ correlation coefficient between images $X$ and $Y$ is defined as follows:

$$
\operatorname{Corr}(X, Y)=\frac{\sum_{i} \sum_{j}\left(X_{i j}-\bar{X}\right)\left(Y_{i j}-\bar{Y}\right)}{\sqrt{\left(\sum_{i} \sum_{j}\left(X_{i j}-\bar{X}\right)^{2}\right)\left(\sum_{i} \sum_{j}\left(Y_{i j}-\bar{Y}\right)^{2}\right)}}
$$

where $X_{i j}$ and $Y_{i j}$ are the pixel values of images $X$ and $Y$, respectively. $\bar{X}$ and $\bar{Y}$ are the mean values of the pixel values of images $X$ and $Y$, respectively. Then the distance between images $X$ and $Y$ can be easily calculated as follows:

$$
\operatorname{Dist}(X, Y)=1-\operatorname{Corr}(X, Y)
$$




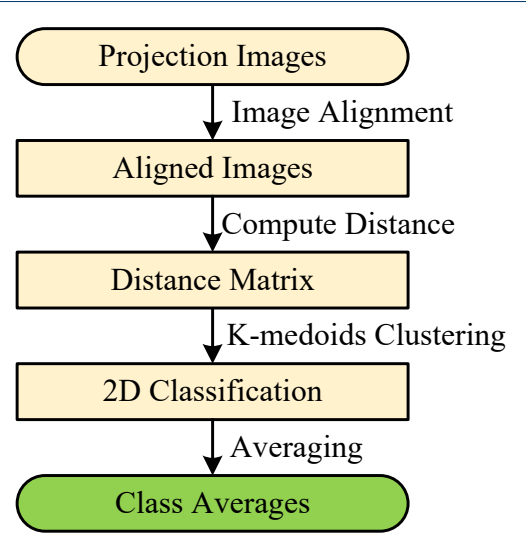

Figure 3 A diagram of the calculation process of the class averaging.

After calculating the distance between all cryo-EM projection images, the distance matrix is used as the input of the K-medoids clustering algorithm for unsupervised 2D classification. Projection images grouped in a class are aligned and averaged to produce a class average. Finally, class averages are used to reconstruct the preliminary 3D model.

\section{Results}

In this section, some simulations are performed to demonstrate the performance of the proposed image alignment algorithm. Firstly, the proposed algorithm is used to estimate alignment parameters. Secondly, the proposed algorithm and the Kmedoids clustering algorithm are used to produce class averages for reconstructing the preliminary $3 \mathrm{D}$ model. The performance of the image alignment algorithm in Fourier space with and without 2D interpolation is compared. The running time of image alignment in Fourier space and real space is also compared. For the convenience of description, in the rest of this paper, the image alignment algorithm in Fourier space using the 2D interpolation is named IAFI, the image alignment algorithm in Fourier space without interpolation is named IAF, and the image alignment algorithm in real space is named IAR. The search step in IAR is 1.

Feasibility of the image alignment algorithm

Here a reference image and a test image are used to estimate alignment parameters of rotation angles and translational shifts in the $\mathrm{x}$-axis and $\mathrm{y}$-axis directions between them. A $256^{*} 256$ Lena image, a simulated $64^{*} 64$ Escherichia coli 50 S ribosomal subunits cryo-EM projection image, and a simulated 128*128 Escherichia coli 70 S ribosomal subunits cryo-EM projection image are chosen as the reference images. Three simulations are designed to test the performance of the proposed algorithm: (1) test images are only rotated; (2) test images are only shifted; and (3) test images are rotated first and then shifted. Fig. 4 shows some test images used in the simulation. All simulations in this subsection are run on MATLAB R2018b on a six-core system with 16-GB RAM in a Windows 10 environment.

The first simulation estimates the rotation angle between the reference image and the test image. The reference image is rotated 100 times randomly in the range of 


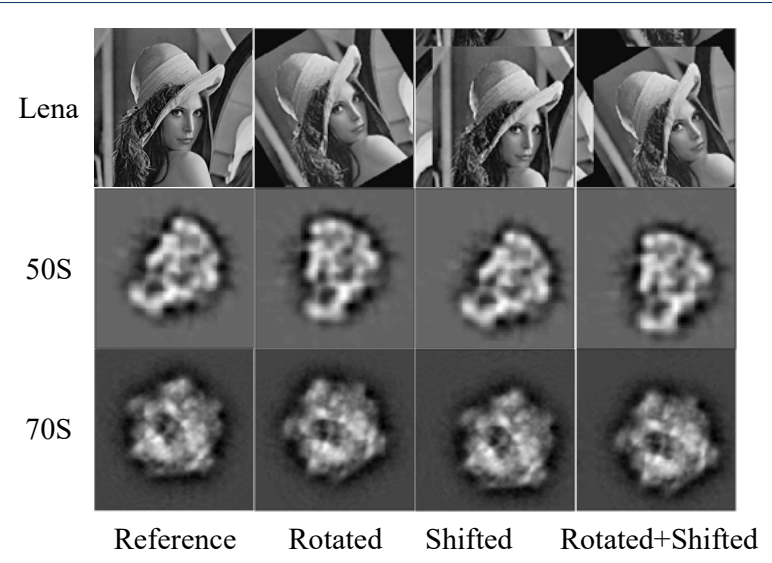

Figure 4 Samples of the test image.

$\left[-180^{\circ}, 180^{\circ}\right]$ to generate 100 test images. The ground truth rotation angles are set to only one decimal place. The rotation angles between images are estimated using the image rotational alignment algorithm described in Section 2.1.

Table 1 shows the frequency distribution of the absolute error in degrees between the estimated and the ground truth rotation angles for different test images. It can be seen that both the IAFI algorithm and the IAF algorithm can estimate the rotation angles with small errors, and the total error of the IAFI algorithm is smaller than that of the IAF algorithm for all test images. It indicates that the proposed image rotational alignment algorithm can estimate the rotation angles between images with high accuracy.

Table 1 The frequency distribution of the absolute error in degrees between the estimated and the ground truth rotation angles for different test images that are only rotated.

\begin{tabular}{|c|c|c|c|c|c|c|}
\hline \multirow{2}{*}{ error } & \multicolumn{2}{|c|}{ Lena } & \multicolumn{2}{|c|}{$50 \mathrm{~S}$} & \multicolumn{2}{|c|}{$70 \mathrm{~S}$} \\
\hline & IAFI & IAF & IAFI & IAF & IAFI & IAF \\
\hline 0 & 48 & 10 & 25 & 4 & 31 & 10 \\
\hline 0.1 & 43 & 20 & 22 & 12 & 68 & 20 \\
\hline 0.2 & 9 & 25 & 4 & 16 & 1 & 25 \\
\hline 0.3 & 0 & 16 & 5 & 10 & 0 & 16 \\
\hline 0.4 & 0 & 13 & 2 & 8 & 0 & 18 \\
\hline 0.5 & 0 & 10 & 6 & 9 & 0 & 10 \\
\hline$>0.5$ & 0 & 6 & 36 & 41 & 0 & 1 \\
\hline $\begin{array}{l}\text { total } \\
\text { error }\end{array}$ & 6.1 & 25.6 & 44 & 59.4 & 7 & 24.6 \\
\hline
\end{tabular}

Table 2 shows the average running time in seconds for different image rotational alignment algorithms to run once. It can be seen that image rotational alignment in Fourier space is much faster than that in real space. In addition, for all of these three algorithms, the larger the image size, the more time they take to rotationally align images. The 2D interpolation calculation in IAFI is very fast, and the estimated rotation angles using IAFI are more accurate than using IAF. It shows that the proposed image rotational alignment algorithm is very efficient.

The second simulation estimates the translational shifts in the $\mathrm{x}$-axis and $\mathrm{y}$-axis directions between the reference image and the test image. The reference image is shifted 100 times randomly in the range of $[-m / 10, m / 10]$ in the $\mathrm{x}$-axis and $\mathrm{y}$-axis directions to generate 100 test images. The ground truth translational shifts are set 
Table 2 The average running time in seconds for different image rotational alignment algorithms to run once for different test images that are only rotated.

\begin{tabular}{ccccc}
\hline images & image size & IAFI & IAF & IAR \\
\hline Lena & $256^{*} 256$ & 0.0094 & 0.0086 & 4.1133 \\
$50 S$ & $64 * 64$ & 0.0048 & 0.0041 & 0.3964 \\
$70 S$ & $128^{*} 128$ & 0.0064 & 0.0056 & 1.0792 \\
\hline
\end{tabular}

to only one decimal place. The translational shifts between images are estimated using the image translational alignment algorithm described in Section 2.2.

Table 3 and Table 4 show the frequency distribution of the absolute error in pixels between the estimated and the ground truth translational shifts in the $\mathrm{x}$-axis and y-axis directions respectively for different test images. It can be seen that the estimated translational shifts using the IAFI algorithm in both the $\mathrm{x}$-axis and $\mathrm{y}$-axis directions for the 50S and 70S cryo-EM projection images are perfectly correct. For the Lena image, the estimated translational shifts using the IAFI algorithm in both the $\mathrm{x}$-axis and $\mathrm{y}$-axis directions are perfectly correct in most cases, and only in a few cases has an error of 0.1. The IAF algorithm can also estimate the translational shifts accurately but has a larger error than the IAFI algorithm. It indicates that the proposed image translational alignment algorithm can also accurately estimate translational shifts between images.

Table 3 The frequency distribution of the absolute error in pixels between the estimated and the ground truth translational shifts in the $x$-axis direction for different test images that are only shifted.

\begin{tabular}{|c|c|c|c|c|c|c|}
\hline \multirow{2}{*}{ error } & \multicolumn{2}{|c|}{ Lena } & \multicolumn{2}{|c|}{$50 \mathrm{~S}$} & \multicolumn{2}{|c|}{$70 \mathrm{~S}$} \\
\hline & IAFI & IAF & IAFI & IAF & IAFI & IAF \\
\hline 0 & 94 & 13 & 100 & 9 & 100 & 9 \\
\hline 0.1 & 6 & 19 & 0 & 23 & 0 & 23 \\
\hline 0.2 & 0 & 15 & 0 & 25 & 0 & 25 \\
\hline 0.3 & 0 & 23 & 0 & 14 & 0 & 17 \\
\hline 0.4 & 0 & 22 & 0 & 21 & 0 & 16 \\
\hline 0.5 & 0 & 8 & 0 & 8 & 0 & 10 \\
\hline$>0.5$ & 0 & 0 & 0 & 0 & 0 & 0 \\
\hline $\begin{array}{l}\text { total } \\
\text { error }\end{array}$ & 0.6 & 24.6 & 0 & 23.9 & 0 & 23.8 \\
\hline
\end{tabular}

Table 4 The frequency distribution of the absolute error in pixels between the estimated and the ground truth translational shifts in the $y$-axis direction for different test images that are only shifted.

\begin{tabular}{|c|c|c|c|c|c|c|}
\hline \multirow{2}{*}{ error } & \multicolumn{2}{|c|}{ Lena } & \multicolumn{2}{|c|}{$50 S$} & \multicolumn{2}{|c|}{$70 S$} \\
\hline & IAFI & IAF & IAFI & IAF & IAFI & IAF \\
\hline 0 & 94 & 5 & 100 & 9 & 100 & 17 \\
\hline 0.1 & 6 & 18 & 0 & 19 & 0 & 21 \\
\hline 0.2 & 0 & 24 & 0 & 17 & 0 & 17 \\
\hline 0.3 & 0 & 21 & 0 & 22 & 0 & 19 \\
\hline 0.4 & 0 & 25 & 0 & 22 & 0 & 20 \\
\hline 0.5 & 0 & 7 & 0 & 11 & 0 & 6 \\
\hline$>0.5$ & 0 & 0 & 0 & 0 & 0 & 0 \\
\hline $\begin{array}{l}\text { total } \\
\text { error }\end{array}$ & 0.6 & 26.4 & 0 & 26.2 & 0 & 22.2 \\
\hline
\end{tabular}

Table 5 shows the average running time in seconds for different image translational alignment algorithms to run once. It can be seen that image translational alignment in Fourier space is much faster than that in real space. Also, for all of these three algorithms, the larger the image size, the more time they take to translationally align images. It shows that the proposed image translational alignment algorithm is very efficient. 
Table 5 The running time in seconds for different image translational alignment algorithms to run once for different test images that are only shifted.

\begin{tabular}{ccccc}
\hline images & image size & IAFI & IAF & IAR \\
\hline Lena & $256 * 256$ & 0.0142 & 0.0133 & 10.8253 \\
$50 \mathrm{~S}$ & $64 * 64$ & 0.0020 & 0.0012 & 0.4982 \\
$70 \mathrm{~S}$ & $128 * 128$ & 0.0043 & 0.0032 & 1.9119 \\
\hline
\end{tabular}

Image alignment with both rotation and translation is more difficult than only rotation or translation. The third simulation estimates the alignment parameters, including rotation angles and translational shifts in the $\mathrm{x}$-axis and $\mathrm{y}$-axis directions between the reference image and the test image. In the single-particle 3D reconstruction, most particles are almost centered in the particle picking procedure, which means only a small number of translational shifts are required. So, a small number of translational shifts are set on the test images in this simulation. The reference image is firstly rotated 100 times randomly in the range of $\left[-180^{\circ}, 180^{\circ}\right]$ and then shifted randomly in the range of $[-m / 20, m / 20]$ in the $\mathrm{x}$-axis and $\mathrm{y}$-axis directions to generate 100 test images. The ground truth rotation angle and translational shifts are set to only one decimal place. The maximum iteration is set as 10.

Table 6 shows the frequency distribution of the absolute error in degrees between the estimated and the ground truth rotation angles for different test images. It can be seen that both the IAFI algorithm and the IAF algorithm can estimate the rotation angle with small errors, and the total error of the IAFI algorithm is smaller than that of the IAF algorithm for all test images. The frequency distribution of the absolute error in pixels between the estimated and the ground truth translational shifts in the $\mathrm{x}$-axis and $\mathrm{y}$-axis directions for different test images are shown in Table 7 and Table 8, respectively. It can be seen that the estimated translational shifts using the IAFI algorithm in both the x-axis and y-axis directions for the 50S and 70S cryo-EM projection images are perfectly correct. For the Lena image, the estimated translational shifts using the IAFI algorithm in both the x-axis and $\mathrm{y}$-axis directions are perfectly correct in most cases, and only in a few cases have a small error. The IAF algorithm can also estimate the translational shifts accurately but has a larger error than the IAFI algorithm. It indicates that the proposed image alignment algorithm is very effective for estimating alignment parameters between images.

Table 6 The frequency distribution of the absolute error in degrees between the estimated and the ground truth rotation angles for different test images that are firstly rotated and then shifted.

\begin{tabular}{|c|c|c|c|c|c|c|}
\hline \multirow{2}{*}{ error } & \multicolumn{2}{|c|}{ Lena } & \multicolumn{2}{|c|}{$50 \mathrm{~S}$} & \multicolumn{2}{|c|}{$70 \mathrm{~S}$} \\
\hline & IAFI & IAF & IAFI & IAF & IAFI & IAF \\
\hline 0 & 22 & 10 & 72 & 10 & 68 & 10 \\
\hline 0.1 & 49 & 14 & 24 & 13 & 25 & 14 \\
\hline 0.2 & 14 & 20 & 2 & 17 & 4 & 20 \\
\hline 0.3 & 7 & 29 & 1 & 18 & 3 & 29 \\
\hline 0.4 & 6 & 18 & 1 & 9 & 0 & 19 \\
\hline 0.5 & 2 & 8 & 0 & 8 & 0 & 8 \\
\hline$>0.5$ & 0 & 1 & 0 & 25 & 0 & 0 \\
\hline $\begin{array}{l}\text { total } \\
\text { error }\end{array}$ & 13.2 & 25.9 & 3.5 & 34.7 & 4.2 & 25.7 \\
\hline
\end{tabular}

Table 9 shows the distribution of the number of the final iterations. It can be seen that both the IAFI algorithm and the IAF algorithm converge within ten iter- 
Table 7 The frequency distribution of the absolute error in pixels between the estimated and the ground truth translational shifts in the $x$-axis direction for different test images that are firstly rotated and then shifted.

\begin{tabular}{|c|c|c|c|c|c|c|}
\hline \multirow{2}{*}{ error } & \multicolumn{2}{|c|}{ Lena } & \multicolumn{2}{|c|}{$50 \mathrm{~S}$} & \multicolumn{2}{|c|}{$70 \mathrm{~S}$} \\
\hline & IAFI & IAF & IAFI & $\mathrm{IAF}$ & IAFI & IAF \\
\hline 0 & 85 & 9 & 100 & 11 & 100 & 8 \\
\hline 0.1 & 15 & 20 & 0 & 19 & 0 & 20 \\
\hline 0.2 & 0 & 23 & 0 & 13 & 0 & 21 \\
\hline 0.3 & 0 & 17 & 0 & 26 & 0 & 27 \\
\hline 0.4 & 0 & 17 & 0 & 18 & 0 & 13 \\
\hline 0.5 & 0 & 11 & 0 & 13 & 0 & 11 \\
\hline$>0.5$ & 0 & 3 & 0 & 0 & 0 & 0 \\
\hline $\begin{array}{l}\text { total } \\
\text { error }\end{array}$ & 1.5 & 25.8 & 0 & 26 & 0 & 25 \\
\hline
\end{tabular}

Table 8 The frequency distribution of the absolute error in pixels between the estimated and the ground truth translational shifts in the $y$-axis direction for different test images that are firstly rotated and then shifted.

\begin{tabular}{|c|c|c|c|c|c|c|}
\hline \multirow{2}{*}{ error } & \multicolumn{2}{|c|}{ Lena } & \multicolumn{2}{|c|}{$50 \mathrm{~S}$} & \multicolumn{2}{|c|}{$70 \mathrm{~S}$} \\
\hline & IAFI & IAF & IAFI & IAF & IAFI & IAF \\
\hline 0 & 63 & 10 & 100 & 13 & 100 & 9 \\
\hline 0.1 & 34 & 24 & 0 & 21 & 0 & 12 \\
\hline 0.2 & 3 & 20 & 0 & 18 & 0 & 19 \\
\hline 0.3 & 0 & 14 & 0 & 21 & 0 & 23 \\
\hline 0.4 & 0 & 13 & 0 & 17 & 0 & 27 \\
\hline 0.5 & 0 & 14 & 0 & 10 & 0 & 10 \\
\hline$>0.5$ & 0 & 5 & 0 & 0 & 0 & 0 \\
\hline $\begin{array}{l}\text { total } \\
\text { error }\end{array}$ & 4 & 25.8 & 0 & 23.8 & 0 & 27.7 \\
\hline
\end{tabular}

ations for all test images. In most cases, the IAFI algorithm and the IAF algorithm require five iterations. On the whole, the proposed image alignment algorithm can accurately align images within ten iterations.

Table 9 The distribution of the number of the final iterations.

\begin{tabular}{|c|c|c|c|c|c|c|}
\hline \multirow{2}{*}{ iterations } & \multicolumn{2}{|c|}{ Lena } & \multicolumn{2}{|c|}{$50 \mathrm{~S}$} & \multicolumn{2}{|c|}{$70 \mathrm{~S}$} \\
\hline & IAFI & IAF & IAFI & IAF & IAFI & IAF \\
\hline 3 & 3 & 9 & 4 & 16 & 6 & 10 \\
\hline 4 & 11 & 36 & 26 & 55 & 13 & 42 \\
\hline 5 & 61 & 42 & 47 & 20 & 74 & 45 \\
\hline 6 & 19 & 12 & 20 & 7 & 7 & 1 \\
\hline 7 & 3 & 1 & 3 & 1 & 0 & 0 \\
\hline 8 & 2 & 0 & 0 & 0 & 0 & 0 \\
\hline 9 & 1 & 0 & 0 & 0 & 0 & 0 \\
\hline 10 & 0 & 0 & 0 & 1 & 0 & 2 \\
\hline $\begin{array}{c}\text { mean } \\
\text { iterations }\end{array}$ & 5.18 & 4.6 & 4.92 & 4.27 & 4.82 & 4.49 \\
\hline
\end{tabular}

\section{Single-particle 3D reconstruction}

The proposed image alignment algorithm and the K-medoids clustering algorithm are used to produce class averages, which are later used for reconstructing the preliminary 3D model. The simulated single-particle cryo-EM projection images of the Escherichia coli $50 \mathrm{~S}$ and $70 \mathrm{~S}$ ribosomal subunit are used in this simulation. The simulated $50 \mathrm{~S}$ and $70 \mathrm{~S}$ projection images are aligned using the proposed image alignment algorithm. The distance matrices for the aligned 50S and 70S cryo-EM projection images are calculated respectively to input to the K-medoids clustering algorithm for computing class averages. The influence of image alignment algorithms IAFI and IAF on 3D reconstruction is compared. This simulation is performed using the ASPIRE software package [49]. 


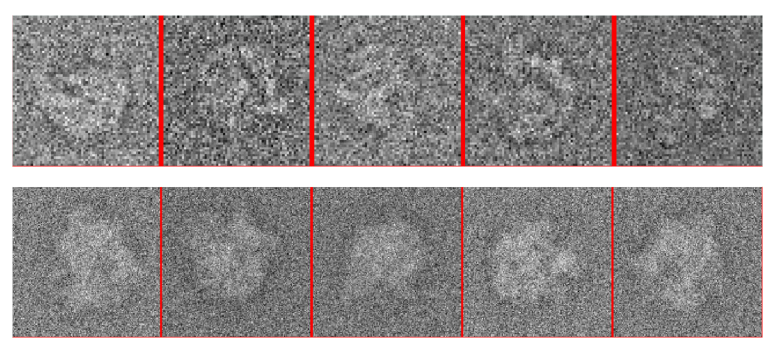

Figure 5 Samples of simulated noisy 50 S (top) and 70 S (bottom) cryo-EM projection images.

The preliminary 3D volume is reconstructed from the generated class averages using the common-lines-based angular reconstruction method [50], which is implemented as the function "cryo_estimate_mean" in the ASPIRE software package. The projection direction of cryo-EM projection images is estimated using the synchronization algorithm [51], where the common lines between projection images are estimated using our proposed weighted voting algorithm [52]. Volumes are rendered using the UCSF Chimera software [53] and filtered using a Gaussian filter with $\sigma=1.0$ pixels.

Firstly, 10000 clean centered 50S projection images with the size of $64 * 64$ pixels and 10000 clean centered $70 \mathrm{~S}$ projection images with the size of $128^{*} 128$ pixels are generated through random rotation matrices corresponding to random projection directions that uniformly distributed over the rotation group $\mathrm{SO}(3)$. The clean centered projection images are shifted randomly in the range of $[-m / 20, m / 20]$ in the $\mathrm{x}$-axis and $\mathrm{y}$-axis directions. The additive Gaussian white noise with the fixed $S N R=0.1$ is added to the clean shifted projection images to generate the final noisy projection images. The $S N R$ is defined as follows:

$$
S N R=\frac{\operatorname{var}(\text { signal })}{\operatorname{var}(\text { noise })}
$$

where var is the variance (energy), signal is the clean projection image, and noise is the noise realization of that projection image. Fig. 5 shows some simulated noisy $50 \mathrm{~S}$ and $70 \mathrm{~S}$ cryo-EM projection images.

Then the simulated noisy 50S and 70S cryo-EM projection images are aligned using the image alignment algorithms IAFI and IAF to compute distance matrices, which are input to the K-medoids clustering algorithm for computing class averages. In the $2 \mathrm{D}$ class averaging, cryo-EM projection images with similar projection directions are classified into one class. The 10000 aligned projection images are classified into 100 classes. Projections classified into one class are aligned and averaged to produce a class average. Fig. 6 and Fig. 7 show some class averages of the $50 \mathrm{~S}$ and $70 \mathrm{~S}$ cryo-EM projection images, respectively, where the image alignment algorithms IAFI and IAF are used.

Finally, the preliminary 3D volume is reconstructed from the class averages. Fig. 8 shows the reconstructed volumes of Escherichia coli 50S (top) and 70S (bottom) ribosomal subunit. The reference volumes are shown on the left side of Fig. 8. The volumes that shown in the middle of Fig. 8 are reconstructed from class averages 


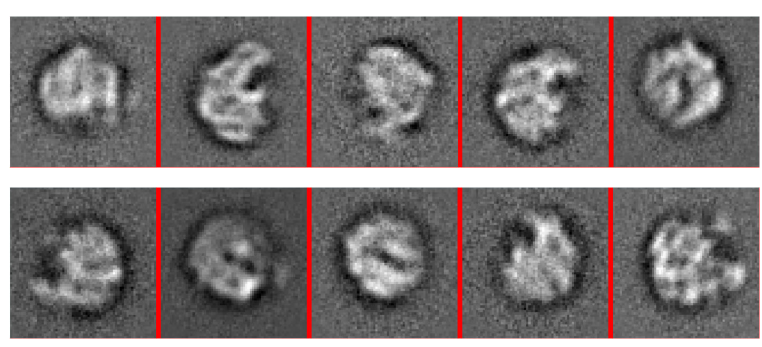

Figure 6 Class averages of 50S cryo-EM projection images. Top: projection images are aligned using the IAFI algorithm. Bottom: projection images are aligned using the IAF algorithm.

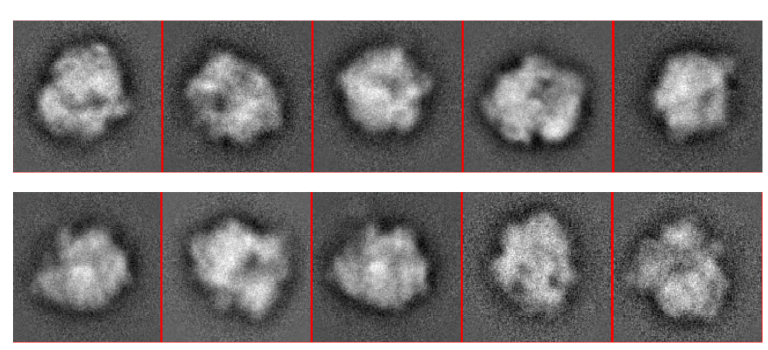

Figure 7 Class averages of $70 \mathrm{~S}$ cryo-EM projection images. Top: projection images are aligned using the IAFI algorithm. Bottom: projection images are aligned using the IAF algorithm.

where the projection images are aligned using the IAFI algorithm. The volumes that shown on the right side of Fig. 8 are reconstructed from class averages where the projection images are aligned using the IAF algorithm. It can be seen that the reconstructed volumes with the IAFI algorithm are more similar to the reference volumes than using the IAF algorithm.

Fig. 9 shows the corresponding Fourier shell correlation (FSC) curves [54] of the reconstructed preliminary 3D volumes of Escherichia coli 50S (Fig. 9(a)) and 70S (Fig. 9(b)) ribosomal subunit for different image alignment algorithms. These FSC curves are computed against the reference volumes shown in Fig. 8. It can be seen that using the IAFI algorithm can obtain better reconstruction resolution than using the IAF algorithm. It indicates that the well-aligned cryo-EM projection images can improve the accuracy of the $2 \mathrm{D}$ classification and further improve the resolution of the preliminary $3 \mathrm{D}$ volume.

\section{Conclusions}

The class averaging technique is useful for the 2D analysis of electron micrographs as well as in single-particle cryo-EM 3D reconstruction. Image alignment is a fundamental step in the class averaging procedure. In this paper, an efficient image alignment algorithm using the $2 \mathrm{D}$ interpolation in the frequency domain is introduced. The proposed image alignment algorithm is tested on a Lena image and two simulated cryo-EM projection images of Escherichia coli 50S and 70S ribosomal subunit for estimating alignment parameters. Simulation results show that the image alignment algorithm in Fourier space using the 2D interpolation can achieve higher estimation accuracy for alignment parameters than the image alignment algorithm in Fourier space without interpolation. It is also found that image alignment in 

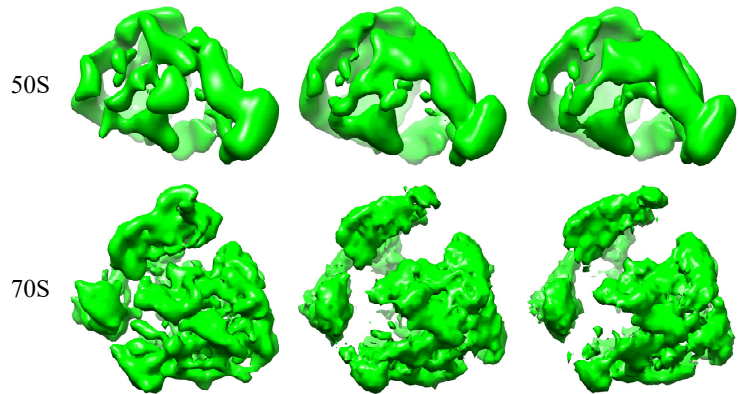

IAFI

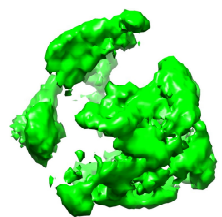

IAF

Figure 8 Reconstructed volumes of Escherichia coli 50S (top) and 70S (bottom) ribosomal subunit with different image alignment algorithms. Left: reference volumes. Middle: reconstructed preliminary volumes from class averages where the projection images are aligned using the IAFI algorithm. Right: reconstructed preliminary volumes from class averages where the projection images are aligned using the IAF algorithm.

Fourier space is faster than that in real space. In addition, the proposed image alignment algorithm and the K-medoids clustering algorithm are used to produce class averages for reconstructing initial 3D volume. Results indicate that the proposed method can be used to improve the resolution of the reconstructed initial model. The main drawback of this study is that the CTF parameters are not considered during the class averaging, which may limit the potential benefits of using the proposed method. In the future work, we will integrate the CTF parameters into the class averaging procedure and apply the method to the real cryo-EM projection images.

\section{Acknowledgements}

None.

Funding

This work was supported by the National Key Research and Development Program of China (2017YFE0111900 and 2018YFB1003205) and the Youth Science and Technology Fund Project of Gansu Province (20JR10RA097).

Abbreviations

2D: two-dimensional; 3D: three-dimensional; cryo-EM: cryo-electron microscopy; SNR: signal-to-noise ratio; FFT: fast Fourier transform; PFFT: polar fast Fourier transform; IAFI: image alignment algorithm in Fourier space using the 2D interpolation; IAF: image alignment algorithm in Fourier space without interpolation; IAR: image alignment algorithm in real space; FSC: Fourier shell correlation.

Availability of data and materials

The simulated data used for benchmarking can be made available upon request from the corresponding author.

Ethics approval and consent to participate

Not applicable.

Competing interests

The authors declare that they have no competing interests.

Consent for publication

Not applicable.

Authors' contributions

$\mathrm{XW}$ wrote the manuscript and performed the simulations. $\mathrm{YL}$ conceived the theory and revised the manuscript. JL contributed to the design of the software. All authors have read and approved the final manuscript.

Author details

${ }^{1}$ School of Information Science and Engineering, Lanzhou University, 730000, Lanzhou, China. ${ }^{2}$ College of

Computer Science and Engineering, Northwest Normal University, 730070, Lanzhou, China. 

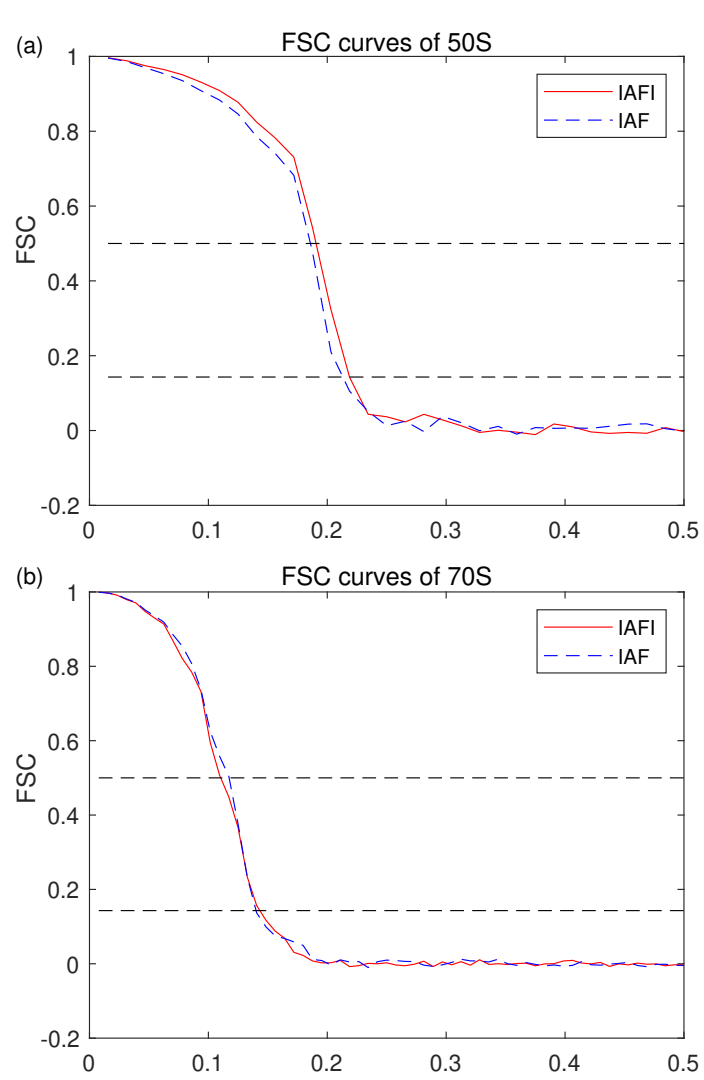

Figure 9 Fourier shell correlation curves of the reconstructed volumes of Escherichia coli 50S (a) and $70 \mathrm{~S}$ (b) ribosomal subunit for image alignment algorithms IAFI and IAF.

\section{References}

1. Bai, X., McMullan, G., Scheres, S.H.W.: How cryo-EM is revolutionizing structural biology. Trends in Biochemical Sciences 40(1), 49-57 (2015)

2. Cheng, Y., Glaeser, R.M., Nogales, E.: How cryo-EM became so hot. Cell 171(6), 1229-1231 (2017)

3. Cheng, Y.: Membrane protein structural biology in the era of single particle cryo-EM. Current Opinion in Structural Biology 52, 58-63 (2018)

4. Earl, L.A., Falconieri, V., Milne, J.L.S., Subramaniam, S.: Cryo-EM: beyond the microscope. Current Opinion in Structural Biology 46, 71-78 (2017)

5. Bendory, T., Bartesaghi, A., Singer, A.: Single-particle cryo-electron microscopy: Mathematical theory, computational challenges, and opportunities. IEEE Signal Processing Magazine 37(2), $58-76$ (2020)

6. Bai, X.: Seeing atoms by single-particle Cryo-EM. Trends in Biochemical Sciences 46(4), 253-254 (2021)

7. Nogales, E., Scheres, S.H.W.: Cryo-EM: a unique tool for the visualization of macromolecular complexity. Molecular Cell 58(4), 677-689 (2015)

8. Nygaard, R., Kim, J., Mancia, F.: Cryo-electron microscopy analysis of small membrane proteins. Current Opinion in Structural Biology 464, 26-33 (2020)

9. Vinothkumar, K.R., Henderson, R.: Single particle electron cryomicroscopy: trends, issues and future perspective. Quarterly Reviews of Biophysics 49, 13 (2016)

10. Danev, R., Yanagisawa, H., Kikkawa, M.: Cryo-electron microscopy methodology: current aspects and future directions. Trends in Biochemical Sciences 44(10), 837-848 (2019)

11. Nogales, E.: The development of cryo-EM into a mainstream structural biology technique. Nature Methods 13(1), 24-27 (2016)

12. Frank, J.: Advances in the field of single-particle cryo-electron microscopy over the last decade. Nature Protocols 12(2), 209-212 (2017)

13. Cheng, Y.: Single-particle cryo-EM - how did it get here and where will it go. Science 361(6405), 876-880 (2018)

14. Nakane, T., Kotecha, A., Sente, A., et al.: Single-particle cryo-EM at atomic resolution. Nature 587(7832), 152-156 (2020)

15. Yip, K.M., Fischer, N., Paknia, E., Chari, A., Stark, H.: Atomic-resolution protein structure determination by cryo-EM. Nature 587(7832), 157-161 (2020)

16. Carazo, J.M., Sorzano, C.O.S., Otón, J., Marabini, R., Vargas, J.: Three-dimensional reconstruction methods in 
single particle analysis from transmission electron microscopy data. Archives of Biochemistry and Biophysics 581, 39-48 (2015)

17. Vilas, J.L., Tabassum, N., Mota, J., Maluenda, D., Jiménez-Moreno, A., Majtner, T., Carazo, J.M., Acton, S.T., Sorzano, C.O.S.: Advances in image processing for single-particle analysis by electron cryomicroscopy and challenges ahead. Current Opinion in Structural Biology 52, 127-145 (2018)

18. Alnabati, E., Kihara, D.: Advances in structure modeling methods for cryo-electron microscopy maps. Molecules 25(1), 82 (2020)

19. Sorzano, C.O.S., Semchonok, D., Lin, S.C., et al.: Algorithmic robustness to preferred orientations in single particle analysis by CryoEM. Journal of Structural Biology 213(1), 107695 (2021)

20. Lyumkis, D.: Challenges and opportunities in cryo-EM single-particle analysis. Journal of Biological Chemistry 294(13), 5181-5197 (2019)

21. Armache, J.-P., Cheng, Y.: Single-particle cryo-EM: beyond the resolution. National Science Review 6(5), 864-866 (2019)

22. Chung, S.-C., Lin, H.-H., Niu, P.-Y., Huang, S.-H., Tu, I.-P., Chang, W.-H.: Pre-pro is a fast pre-processor for single-particle cryo-EM by enhancing 2D classification. Communications Biology 3, 508 (2020)

23. Park, W., Madden, D.R., Rockmore, D.N., Chirikjian, G.S.: Deblurring of class-averaged images in single-particle electron microscopy. Inverse Problems 26(3), 035002 (2010)

24. Park, W., Midgett, C.R., Madden, D.R., Chirikjian, G.S.: A stochastic kinematic model of class averaging in single-particle electron microscopy. The International Journal of Robotics Research 30(6), 730-754 (2011)

25. Singer, A., Sigworth, F.J.: Computational methods for single-particle electron cryomicroscopy. Annual Review of Biomedical Data Science 3, 163-190 (2020)

26. Joyeux, L., Penczek, P.A.: Efficiency of 2D alignment methods. Ultramicroscopy 92(2), 33-46 (2002)

27. Yang, Z., Penczek, P.A.: Cryo-EM image alignment based on nonuniform fast Fourier transform. Ultramicroscopy 108(9), 959-969 (2008)

28. Gallagher, J.R., Kim, A.J., Gulati, N.M., Harris, A.K.: Negative-stain transmission electron microscopy of molecular complexes for image analysis by 2D class averaging. Current Protocols in Microbiology 54(1), 90 (2019)

29. Yin, S., Zhang, B., Yang, Y., Huang, Y., Shen, H.: Clustering enhancement of noisy cryo-electron microscopy single-particle images with a network structural similarity metric. Journal of Chemical Information and Modeling 59(4), 1658-1667 (2019)

30. Sorzano, C.O.S., Bilbao-Castro, J.R., Shkolnisky, Y., Alcorlo, M., Melero, R., Caffarena-Fernández, G., Li, M., Xue, G., Marabini, R., Carazo, J.M.: A clustering approach to multireference alignment of single-particle projections in electron microscopy. Journal of Structural Biology 171(2), 197-206 (2010)

31. van Heel, M., Frank, J.: Use of multivariates statistics in analysing the images of biological macromolecules. Ultramicroscopy 6(1), 187-194 (1981)

32. Penczek, P., Radermacher, M., Frank, J.: Three-dimensional reconstruction of single particles embedded in ice. Ultramicroscopy 40(1), 33-53 (1992)

33. Zhao, Z., Singer, A.: Rotationally invariant image representation for viewing direction classification in cryo-EM. Journal of Structural Biology 186(1), 153-166 (2014)

34. Reboul, C.F., Bonnet, F., Elmlund, D., Elmlund, H.: A stochastic hill climbing approach for simultaneous 2D alignment and clustering of cryogenic electron microscopy images. Structure 24(6), 988-996 (2016)

35. Bhamre, T., Zhao, Z., Singer, A.: Mahalanobis Distance for Class Averaging of cryo-EM Images. In: 2017 IEEE 14th International Symposium on Biomedical Imaging (ISBI 2017), pp. 654-658 (2017)

36. Ma, C., Bendory, T., Boumal, N., Sigworth, F., Singer, A.: Heterogeneous multireference alignment for images with application to $2 \mathrm{D}$ classification in single particle reconstruction. IEEE Transactions on Image Processing 29, 1699-1710 (2020)

37. Marabini, R., Masegosa, I.M., San Martín, M.C., Marco, S., Fernández, J.J., de la Fraga, L.G., Vaquerizo, C., Carazo, J.M.: Xmipp: an image processing package for electron microscopy. Journal of Structural Biology 116(1), 237-240 (1996)

38. Sorzano, C.O.S., Marabini, R., Velázquez-Muriel, J., Bilbao-Castro, J.R., Scheres, S.H.W., Carazo, J.M., Pascual-Montano, A.: Xmipp: a new generation of an open-source image processing package for electron microscopy. Journal of Structural Biology 148(2), 194-204 (2004)

39. de la Rosa-Trevín, J.M., Otón, J., Marabini, R., Zaldívar, A., Vargas, J., Carazo, J.M., Sorzano, C.O.S.: Xmipp 3.0: an improved software suite for image processing in electron microscopy. Journal of Structural Biology 184(2), 321-328 (2013)

40. Ludtke, S.J., Baldwin, P.R., Chiu, W.: EMAN: semiautomated software for high-resolution single-particle reconstructions. Journal of Structural Biology 128(1), 82-97 (1999)

41. Tang, G., Peng, L., Baldwin, P.R., Mann, D.S., Jiang, W., Rees, I., Ludtke, S.J.: EMAN2: an extensible image processing suite for electron microscopy. Journal of Structural Biology 157(1), 38-46 (2007)

42. Punjani, A., Rubinstein, J.L., Fleet, D.J., Brubaker, M.A.: cryoSPARC: algorithms for rapid unsupervised cryo-EM structure determination. Nature Methods 14(3), 290-296 (2017)

43. Grant, T., Rohou, A., Grigorieff, N.: cisTEM, user-friendly software for single-particle image processing. eLife 7 35383 (2018)

44. Scheres, S.H.W.: RELION: Implementation of a Bayesian approach to cryo-EM structure determination. Journal of Structural Biology 180(3), 519-530 (2012)

45. Kimanius, D., Forsberg, B.O., Scheres, S.H.W., Lindahl, E.: Accelerated cryo-EM structure determination with parallelisation using GPUs in RELION-2. eLife 5, 18722 (2016)

46. Zivanov, J., Nakane, T., Forsberg, B.O., Kimanius, D., Hagen, W.J.H., Lindahl, E., Scheres, S.H.W.: New tools for automated high-resolution cryo-EM structure determination in RELION-3. eLife 7, 42166 (2018)

47. Yang, Z., Fang, J., Chittuluru, J., Asturias, F.J., Penczek, P.A.: Iterative stable alignment and clustering of 2D transmission electron microscope images. Structure 20(2), 237-247 (2012)

48. Park, H.-S., Jun, C.-H.: A simple and fast algorithm for K-medoids clustering. Expert Systems with 
Applications 36(2), 3336-3341 (2009)

49. ASPIRE: ASPIRE-Algorithms for Single Particle Reconstruction software package. http://spr.math.princeton.edu/ (2017)

50. van Heel, M.: Angular reconstitution: a posteriori assignment of projection directions for 3D reconstruction. Ultramicroscopy 21, 111-123 (1987)

51. Shkolnisky, Y., Singer, A.: Viewing direction estimation in cryo-EM using synchronization. SIAM Journal on Imaging Sciences 5(3), 1088-1110 (2012)

52. Wang, X., Lu, Y., Lu, Z., Ran, X., Liu, J.: A Weighted Voting Algorithm for Detecting Reliable Common Lines in Single Particle Cryo-EM. In: 2019 IEEE International Conference on Bioinformatics and Biomedicine (BIBM 2019), pp. 98-101 (2019)

53. Pettersen, E.F., Goddard, T.D., Huang, C.C., Couch, G.S., Greenblatt, D.M., Meng, E.C., Ferrin, T.E.: UCSF Chimera-a visualization system for exploratory research and analysis. Journal of Computational Chemistry 25(13), 1605-1612 (2004)

54. van Heel, M., Schatz, M.: Fourier shell correlation threshold criteria. Journal of Structural Biology 151(3), 250-262 (2005) 
Figures

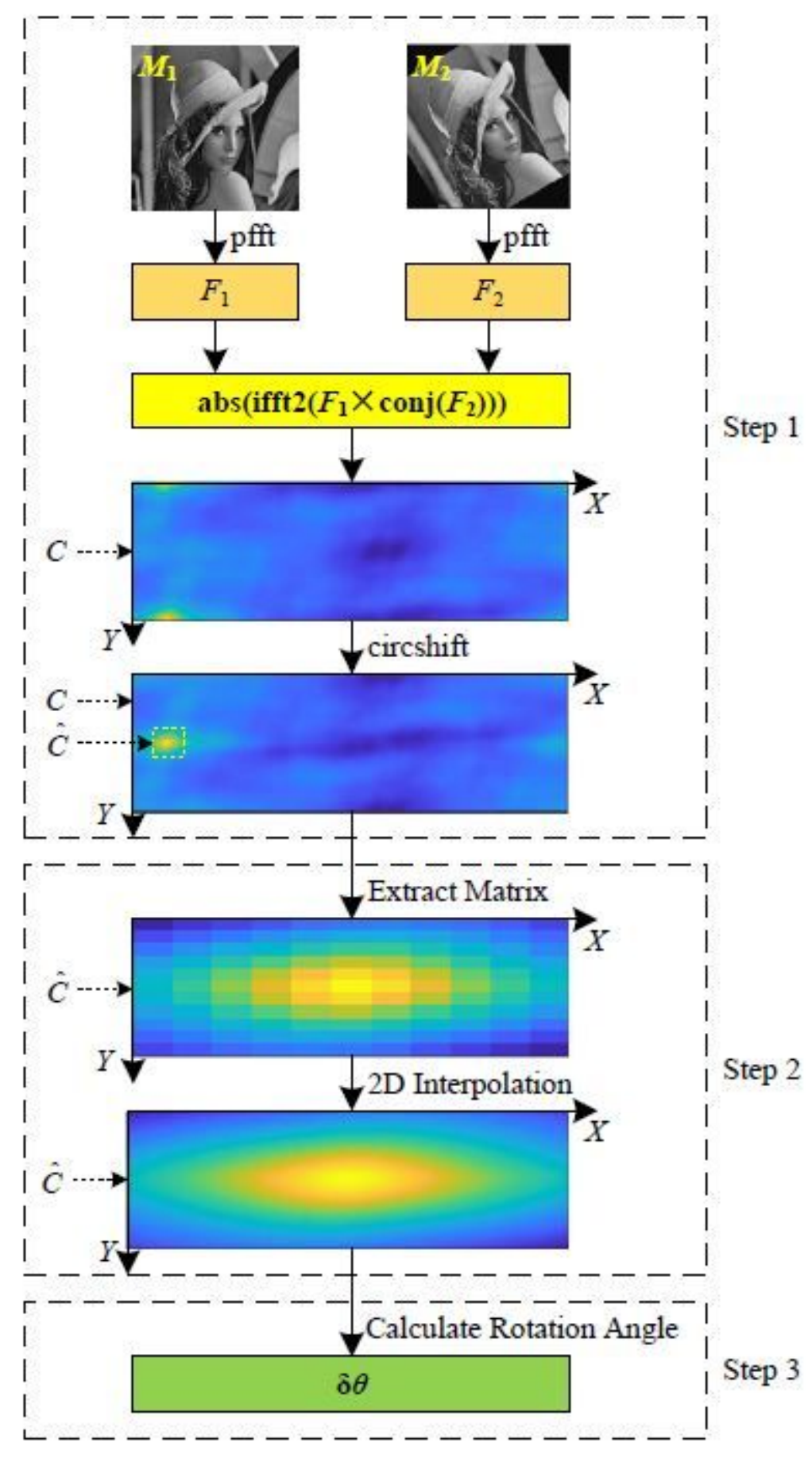

Figure 1

A diagram of the proposed image rotational alignment algorithm using 2D interpolation in the frequency domain of images. 


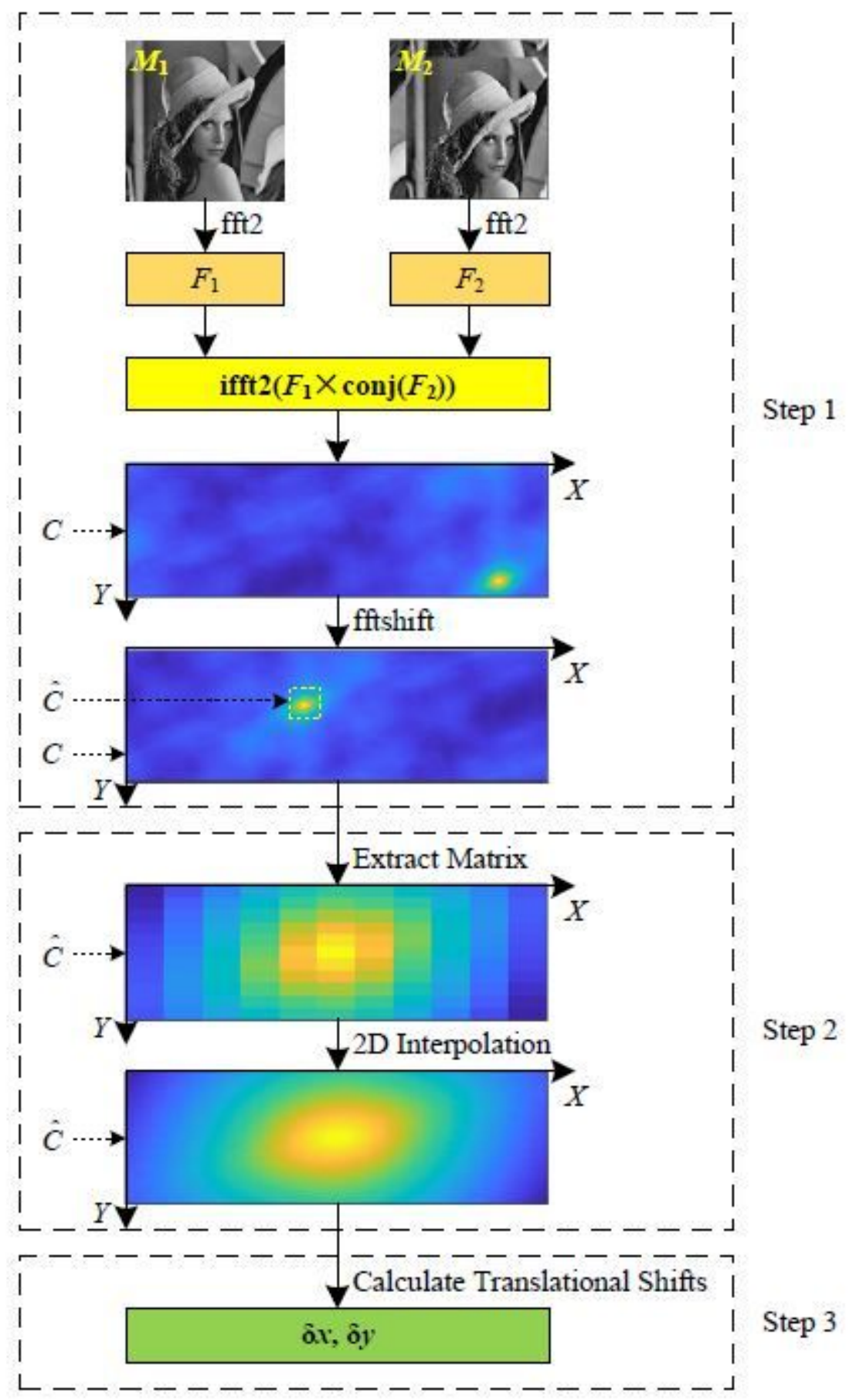

Figure 2

A diagram of the proposed image translational alignment algorithm using 2D interpolation in the frequency domain of images. 


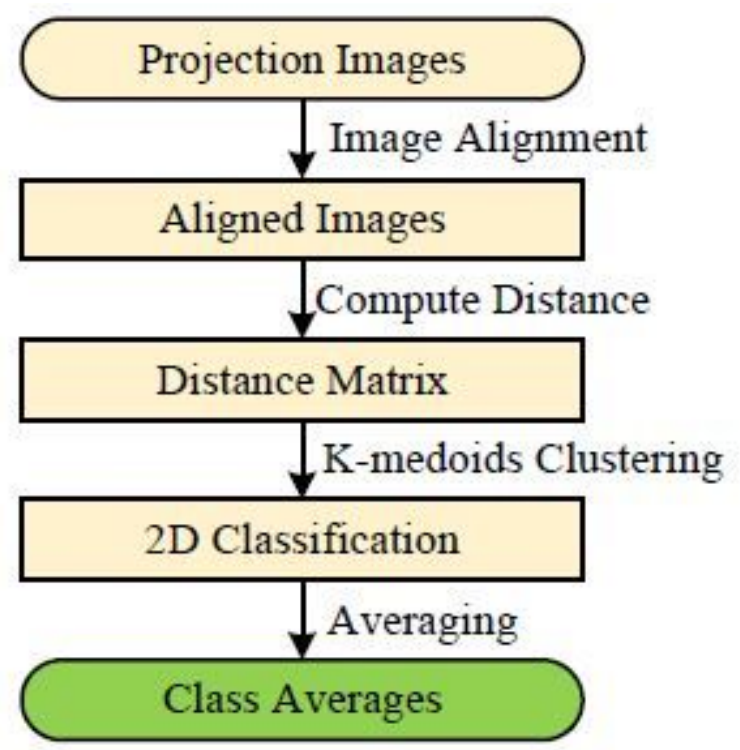

Figure 3

A diagram of the calculation process of the class averaging.

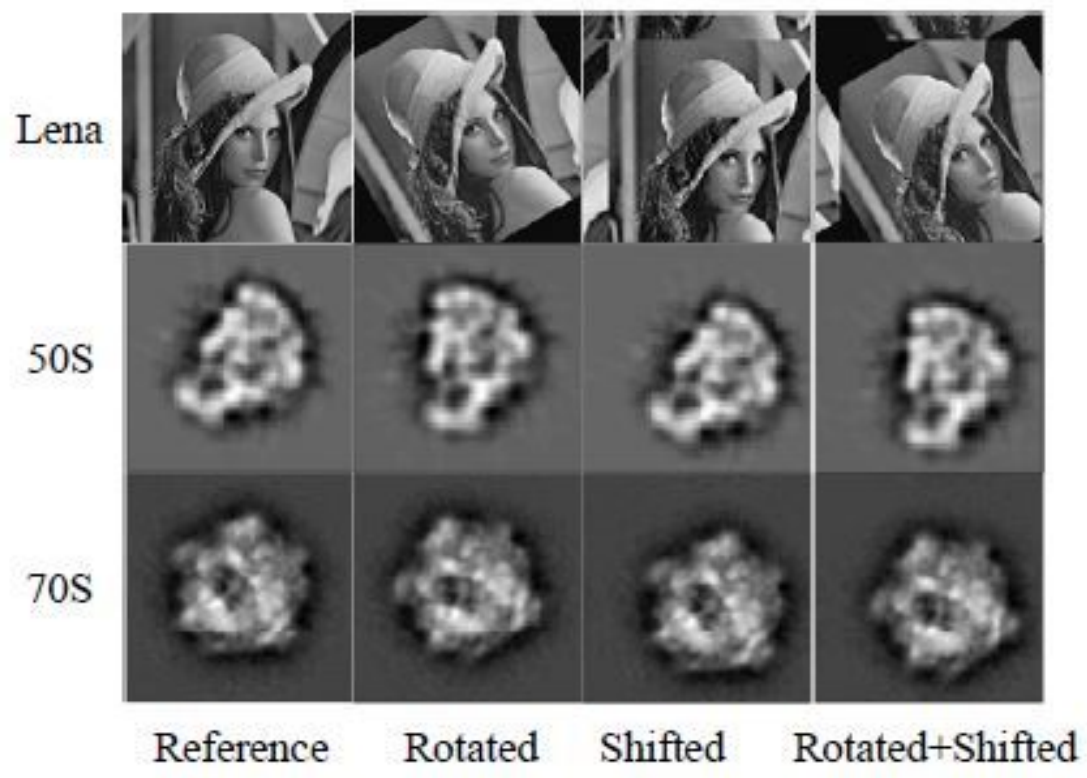

Figure 4

Samples of the test image. 

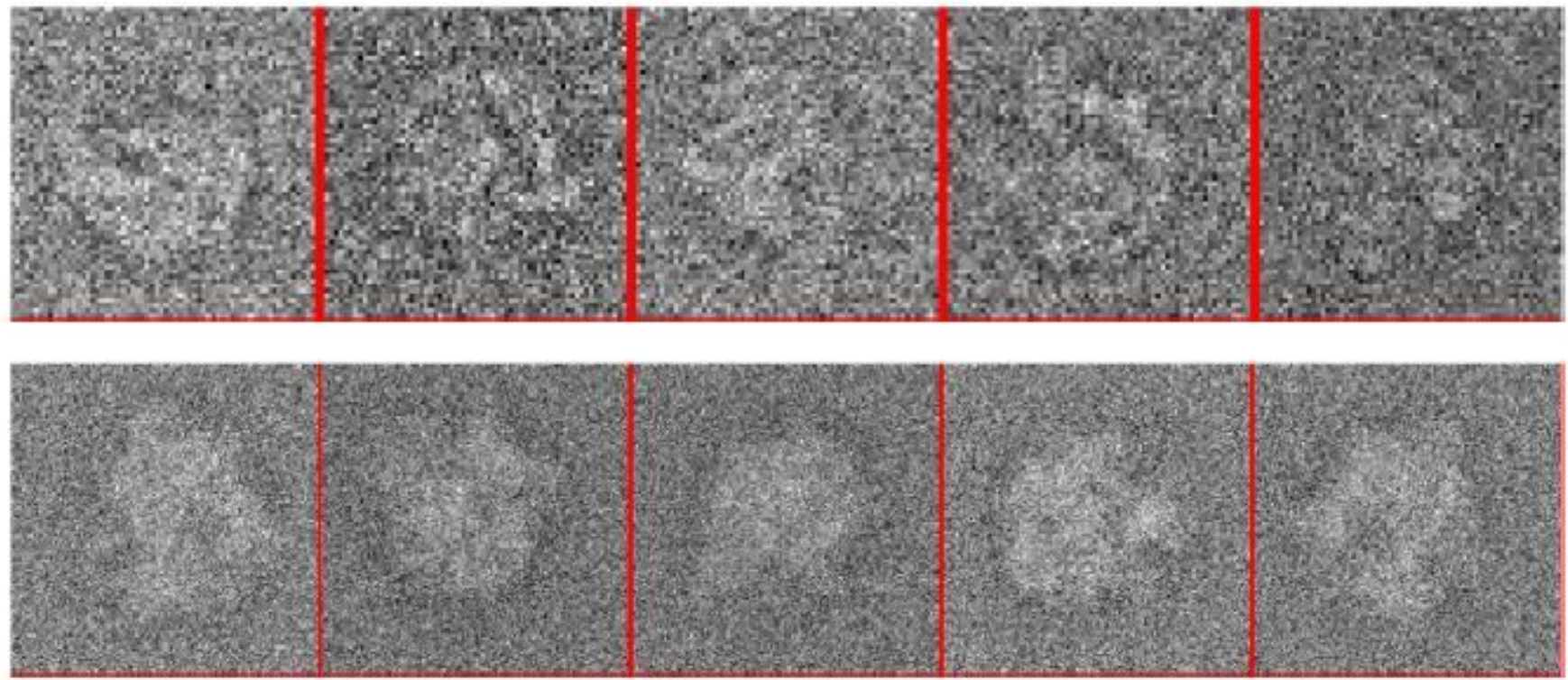

\section{Figure 5}

Samples of simulated noisy 50S (top) and 70S (bottom) cryo-EM projection images.
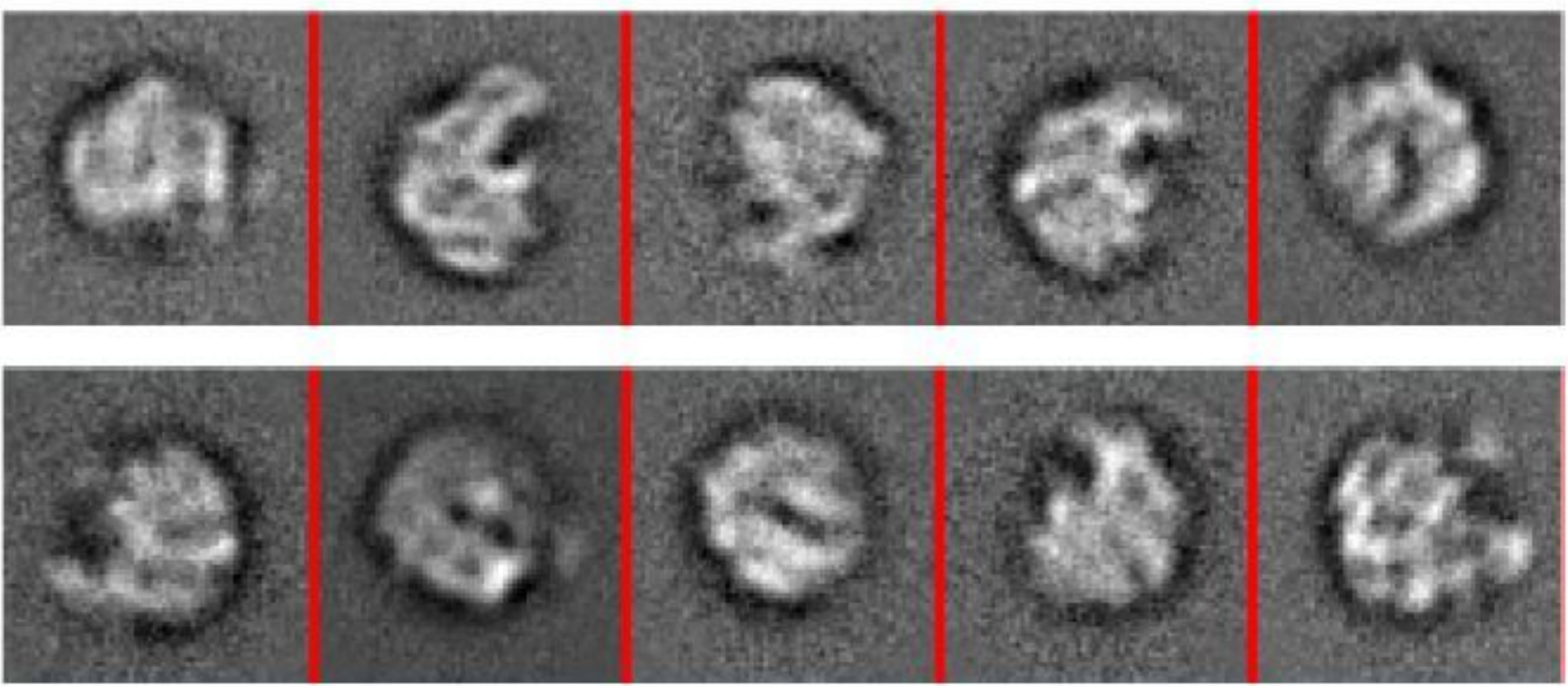

\section{Figure 6}

Class averages of 50S cryo-EM projection images. Top: projection images are aligned using the IAFI algorithm. Bottom: projection images are aligned using the IAF algorithm. 

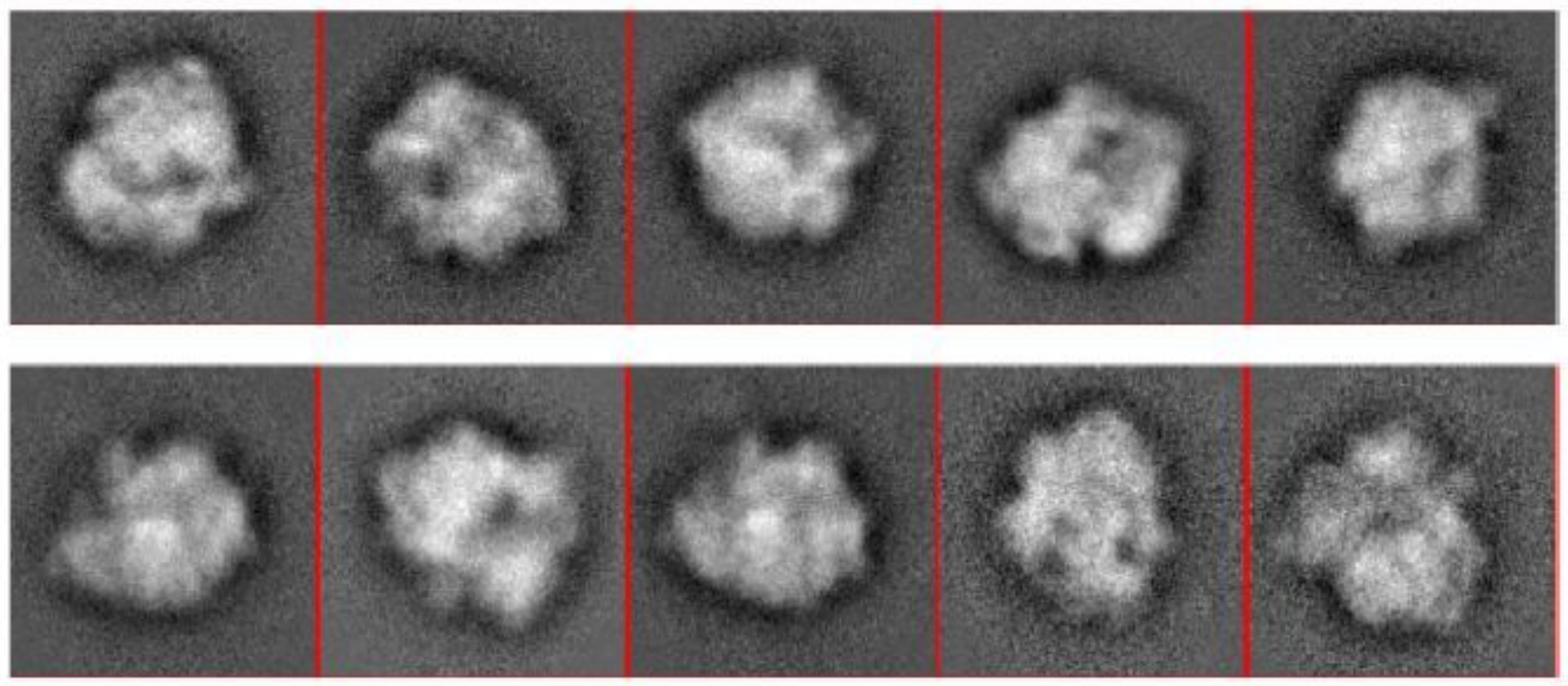

Figure 7

Class averages of 70S cryo-EM projection images. Top: projection images are aligned using the IAFI algorithm. Bottom: projection images are aligned using the IAF algorithm.

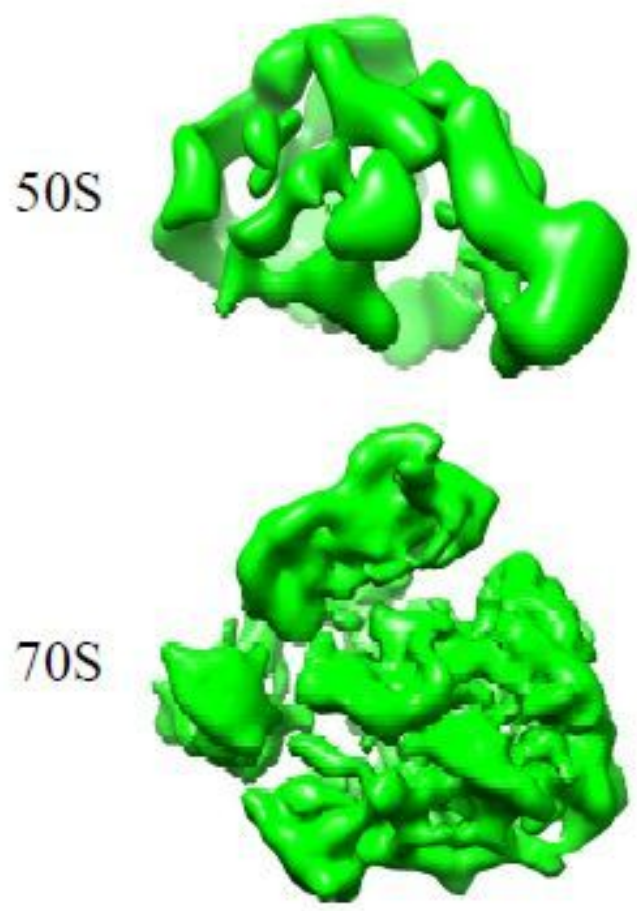

Reference
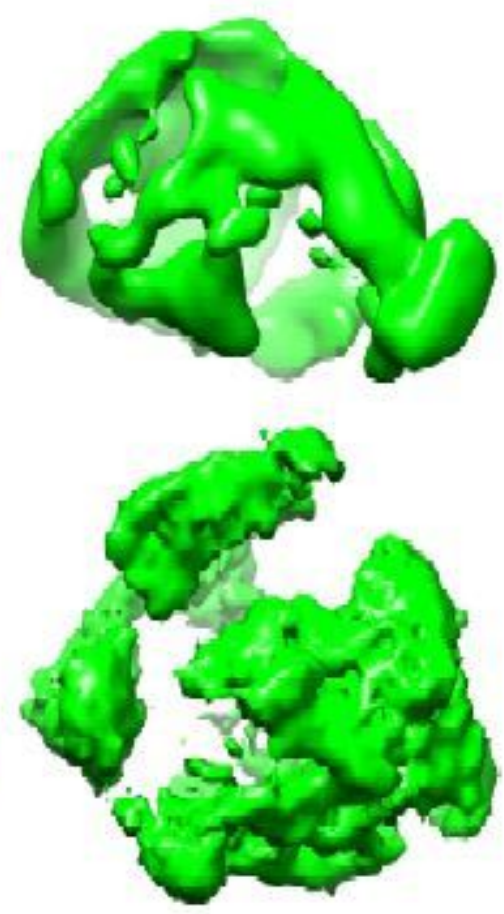

IAFI
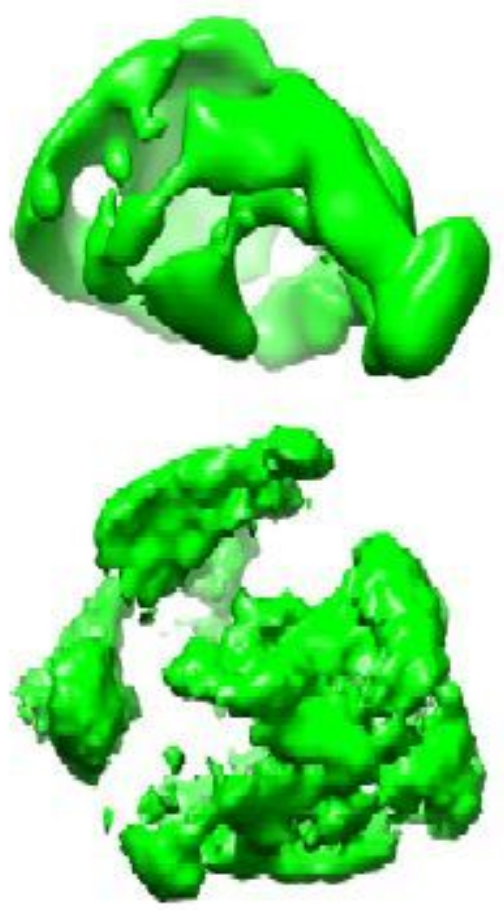

IAF

\section{Figure 8}

Reconstructed volumes of Escherichia coli 50 S (top) and 70S (bottom) ribosomal subunit with different image alignment algorithms. Left: reference volumes. Middle: reconstructed preliminary volumes from 
class averages where the projection images are aligned using the IAFI algorithm. Right: reconstructed preliminary volumes from class averages where the projection images are aligned using the IAF algorithm.
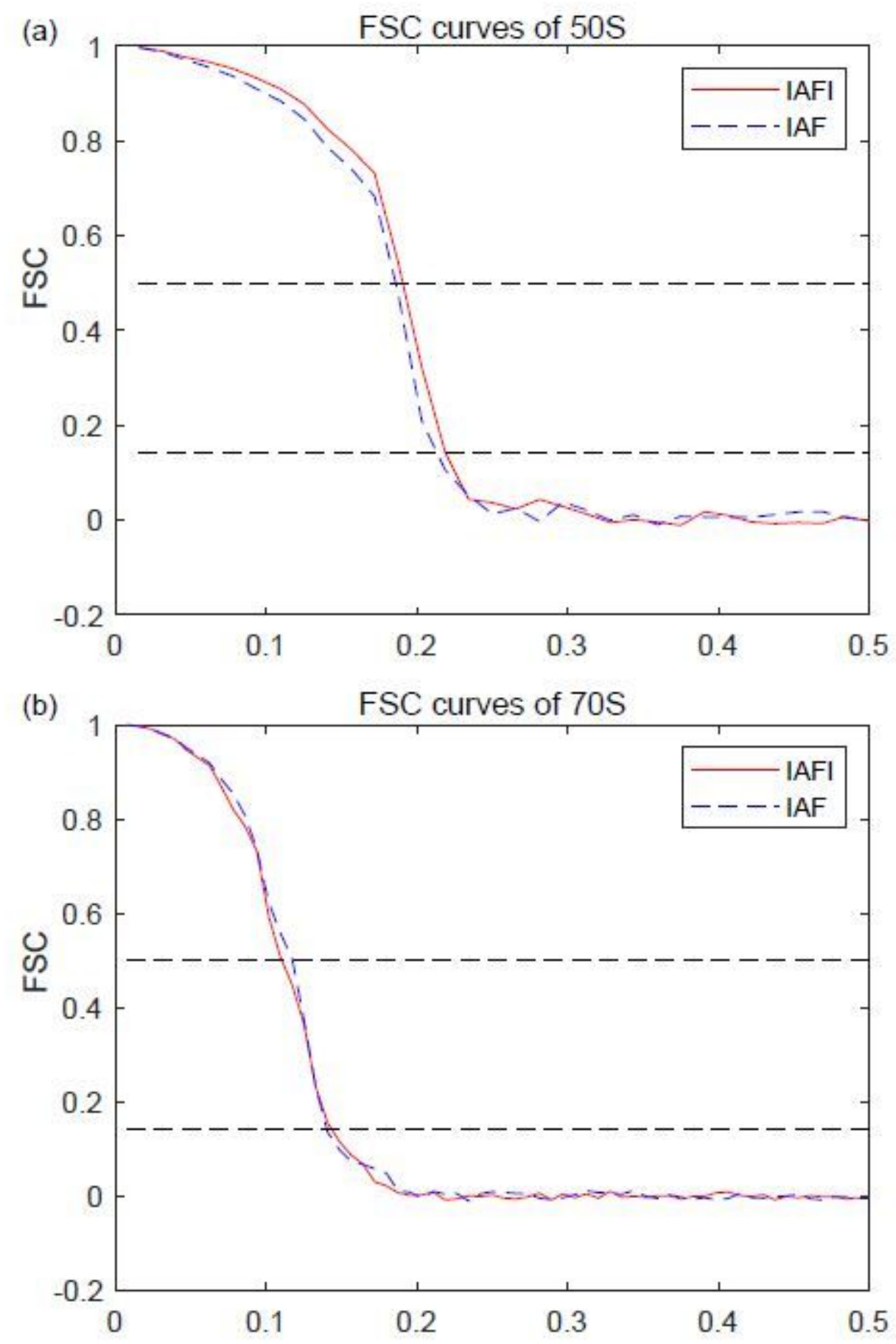

\section{Figure 9}

Fourier shell correlation curves of the reconstructed volumes of Escherichia coli 50S (a) and 70S (b) ribosomal subunit for image alignment algorithms IAFI and IAF. 